\title{
La terapia cognitivo conductual y los síntomas negativos en la esquizofrenia
}

\author{
Tania Morales Vigil, ${ }^{1}$ Ana Fresán Orellana, ${ }^{2}$ Rebeca Robles García, ${ }^{2}$ Mariana Domínguez Correa ${ }^{3}$
}

Actualización por temas

\section{ABSTRACT}

\section{Introduction}

Schizophrenia is a health and social problem of great dimensions, affecting not only the patient but their family and social environment. Within the psychotherapeutic approaches for patients with schizophrenia, Cognitive Behavioral Therapy (CBT) has most evidence of effectiveness for different porpoises and stages of the disorder.

\section{Objective}

Analyze the specific techniques and outcome on negative symptoms of the major CBT for patients with schizophrenia: Psychoeducation, Social Skills Training, Cognitive Remediation, CBT for positive symptoms and Cognitive Behavioral Recovery Oriented Therapy (CBT-R).

\section{Method}

A searching and analysis of scientific literature published in English and Spanish between 1990 and 2014 were performed in PSYCLIT, MEDLINE, EBSCO-HOST and PROQUEST, employing as key words the names of the different CBT for schizophrenia and the diagnosis. Relevant literature included in review articles was also included.

\section{Results}

Despite the advances of CBT, the problems generated by negative symptoms of patients with schizophrenia are not completely solved.

\section{Discussion and conclusion}

CBT-R is considered a promising therapeutic style to achieve the objectives of recovery in patients with low psychosocial functioning and predominance of negative symptomatology.

Key words: Cognitive Behavioral Therapy, schizophrenia, negative symptoms, recovery.

\section{RESUMEN}

\section{Introducción}

La esquizofrenia constituye un problema sanitario y social de grandes dimensiones que afecta no sólo al paciente sino a su entorno familiar y social. Dentro de las aproximaciones psicoterapéuticas dirigidas a pacientes con esquizofrenia, las terapias cognitivo conductuales (TCC) son las que cuentan con mayor evidencia de efectividad para diferentes propósitos y etapas del trastorno.

\section{Objetivo}

Analizar las técnicas y efectos específicos sobre síntomas negativos de las principales TCC para pacientes con esquizofrenia: Psicoeducación, Entrenamiento en Habilidades Sociales, Rehabilitación Cognitiva, TCC para Síntomas Positivos y TCC orientada a la Recuperación (TCC-R).

\section{Método}

Se llevó a cabo una búsqueda y análisis de literatura científica en PSYCLIT, MEDLINE, EBSCO-HOST y PROQUEST publicada entre 1990 y 2014 en revistas indexadas en inglés y español, utilizando como palabras clave los nombres de las diferentes TCC para la esquizofrenia que se cruzaron en todos los casos con el diagnóstico. Se recopiló también la literatura relevante citada en estos artículos, sobre todo en las revisiones de literatura antecedentes.

\section{Resultados}

A pesar de los avances que han presentado las TCC en la recuperación de pacientes con diagnóstico de esquizofrenia, los problemas generados por la sintomatología negativa no han sido resueltos por completo.

\section{Discusión y conclusión}

La TCC-R se considera un estilo terapéutico prometedor para alcanzar los objetivos de recuperación de los pacientes con bajo funcionamiento psicosocial y predominio de sintomatología negativa.

Palabras clave: Terapia cognitivo conductual, esquizofrenia, síntomas negativos, recuperación.

Facultad de Psicología, Universidad Nacional Autónoma de México, México.

Instituto Nacional de Psiquiatría Ramón de la Fuente Muñiz, México.

Psicología Clínica, Universidad del Valle de México, México.

Correspondencia: Dra. Rebeca Robles García. Dirección de Investigaciones Epidemiológicas y Psicosociales. Instituto Nacional de Psiquiatría Ramón de la Fuente Muñiz. Calz. México-Xochimilco 101, San Lorenzo Huipulco, Tlalpan, 14370, México, DF, E-mail: reberobles@imp.edu.mx

Recibido primera versión: 25 de junio de 2014. Segunda versión: 24 de marzo de 2015. Aceptado: 27 de julio de 2015. 


\section{INTRODUCCIÓN}

La esquizofrenia, un padecimiento de baja incidencia pero con gran discapacidad asociada, se caracteriza por la presencia de tres grandes grupos de síntomas: los síntomas positivos -resultado de procesos mentales anormales (alucinaciones y delirios)-, los síntomas negativos -la disminución/ ausencia de funcionamiento mental normal (aplanamiento afectivo, aislamiento, disminución de la motivación o energía y anhedonia)-, y los síntomas desorganizados relativos a la desorganización en el pensamiento, lenguaje o comportamiento (circunstancialidad, tangencialidad, descarrilamiento, "ensalada" de palabras, lentitud psicomotriz, movimientos o gestos rítmicos y repetitivos y conductas extrañas sin un propósito definido).

Afortunadamente, las expectativas del tratamiento del padecimiento se han modificado positivamente a lo largo del tiempo. Se han registrado avances importantes en los tratamientos farmacológicos, que son cada vez más eficaces y selectivos en el manejo y reducción de la sintomatología psicótica positiva. Además, se ha transitado de un modelo institucionalizador a uno basado en la comunidad y orientado a la recuperación. Así, el campo de la rehabilitación psicosocial se ha desarrollado al punto de lograr la oferta de estrategias esenciales para la mejora de la autonomía del paciente y su participación activa dentro de la comunidad.

Es así como los tratamientos psicosociales han evolucionado partiendo de orígenes pesimistas, que contemplaban la desorganización de la personalidad como una consecuencia inevitable del deterioro cognitivo de la psicosis y que por tanto descartaban la posibilidad de un tratamiento psicológico, hasta llegar al desarrollo de múltiples modalidades de intervención encaminadas a la recuperación de los pacientes.

Hoy en día, las estrategias psicosociales se consideran parte sustancial del tratamiento de la esquizofrenia; tanto más que es limitada la efectividad de los fármacos en la mejoría de los síntomas negativos. ${ }^{1}$ Aunque estos síntomas no son tan prominentes como los positivos, constituyen a largo plazo los mejores indicadores de la discapacidad relacionada con la enfermedad, pues interfieren con la capacidad del paciente para funcionar en la vida diaria. ${ }^{2}$

Entre las intervenciones psicosociales más empleadas en el tratamiento de la esquizofrenia destacan, debido a su efectividad, las terapias cognitivo conductuales (TCC). ${ }^{3}$

Se distinguen tres etapas en la evolución de las TCC para la psicosis: en la primera, durante los años 1960, las estrategias terapéuticas se basaron en los principios del condicionamiento operante al dirigirse al control ambiental de la conducta; en la segunda, en la década de 1970, se constató la introducción de los tratamientos familiares y de los procedimientos de entrenamiento en habilidades sociales; y en la tercera, a partir de los años 1990, se consolidaron estas dos modalidades previas de intervención, desarrollándose así lo que se conoce a la fecha como la TCC para la esquizofrenia. ${ }^{4}$
Las técnicas de intervención de corte cognitivo-conductual están demostrando cada vez más su eficacia en la prevención de las recaídas ${ }^{5}$ y en el manejo de problemas $^{6}$ de pacientes con esquizofrenia; promueven su recuperación e integración a la comunidad debido a que se dirigen hacia componentes clave para la funcionalidad como la estabilidad sintomática, la promoción de una vida independiente, el establecimiento laboral y el adecuado funcionamiento social..$^{5-7}$

Distintos autores han coincidido en que la TCC para la esquizofrenia puede dividirse en cuatro categorías:3,5-8 la terapia familiar psicoeducativa, ${ }^{9}$ el entrenamiento en habilidades sociales y en la solución de problemas, ${ }^{10}$ las terapias de rehabilitación cognitiva ${ }^{11}$ y la terapia cognitivo-conductual para síntomas positivos. ${ }^{12,13}$ Recientemente se suma a la lista de las principales TCC para la esquizofrenia la terapia cognitivo-conductual orientada a la recuperación (TCC-R), pues ha mostrado resultados sin precedentes, en especial respecto al manejo de los síntomas negativos. ${ }^{14}$

Tomando en cuenta todo lo anterior, el objetivo del presente trabajo fue analizar las técnicas y efectos específicos sobre los síntomas negativos de las principales TCC para pacientes con esquizofrenia (psicoeducación, entrenamiento en habilidades sociales, rehabilitación cognitiva, TCC para síntomas positivos y TCC-R).

\section{MÉTODO}

Se llevó a cabo la búsqueda (en PSYCLIT, MEDLINE, EBSCO-HOST y PROQUEST) así como la recopilación y análisis de la literatura científica publicada entre 1990 y 2014 en revistas indexadas en inglés y español, utilizando como palabras clave los nombres de las diferentes TCC para la esquizofrenia (Psicoeducación, Entrenamiento en Habilidades Sociales, Rehabilitación Cognitiva, TCC para Síntomas Positivos y TCC-R) que se cruzó en todos los casos con el diagnóstico (esquizofrenia). Se complementó con la bibliografía relevante citada en los textos recopilados, sobre todo en las revisiones previas. Se analizó el tipo de variables de resultado de las intervenciones que eran seleccionados en los diferentes estudios como indicadores de efectividad de las intervenciones. Esto permitió determinar si se evaluaba su efecto específico en los síntomas negativos y si esto se hacía con el rigor metodológico necesario.

\section{RESULTADOS}

Se analizaron un total de 37 trabajos empíricos (ocho de ellos meta-análisis) para evaluar la eficacia de las intervenciones en la esquizofrenia, sujetas a estudio (Psicoeducación, Entrenamiento en Habilidades Sociales, Rehabilitación Cognitiva, TCC para Síntomas Positivos y TCC-R); de éstos, únicamente 18 evaluaron su impacto en los síntomas negati- 
vos de los pacientes. El análisis de la literatura especializada se presenta a propósito de cada una de las TCC para la esquizofrenia, ofreciendo primero una breve definición de la técnica y su efectividad general, para concluir con el análisis de su impacto en el manejo de la sintomatología negativa.

\section{Psicoeducación familiar}

La terapia psicoeducativa se fundamenta en las investigaciones que subrayan la relación entre el ambiente familiar, ${ }^{15,16}$ la emotividad expresada de los familiares ${ }^{17}$ y la cohesión familiar ${ }^{18}$ con el curso del padecimiento; dichas intervenciones se dirigen a brindar información sobre éste a los familiares como herramienta para favorecer su relación con el paciente; dentro de las actividades más comunes se encuentran: clarificar el término "esquizofrenia", describir los síntomas (positivos, cognitivos y negativos) y su origen (alteraciones en la dopamina y en el procesamiento de la información), explicar el modelo de vulnerabilidad-estrés, dar a conocer las características de los medicamentos y sus efectos secundarios, así como la posibilidad de la prevención de recaídas con base en un plan de acción en caso de crisis;, ${ }^{19}$ recientemente se han incluido técnicas de entrenamiento en estrategias de afrontamiento y solución de problemas. ${ }^{20}$

La psicoeducación a los familiares es el tipo de terapia más investigada en el caso de la esquizofrenia; ${ }^{21}$ desde la década de 1980 se han realizado estudios respecto a la relación entre la alta emotividad expresada de la familia y el curso del padecimiento, reportando menor tasa de recaídas en los pacientes cuyos familiares recibieron el tratamiento, incluso a un seguimiento a nueve meses ${ }^{22}$ y dos años. ${ }^{23}$

Las intervenciones de psicoeducación combinadas con los medicamentos antipsicóticos han demostrado ser eficaces para incrementar el funcionamiento psicosocial y disminuir la emotividad expresada, ${ }^{3}$ la sintomatología clínica, las recaídas y las hospitalizaciones; ${ }^{22}$ también han demostrado efectividad en variables más indirectas como el mantenimiento del empleo, ${ }^{23}$ se ha reportado que estos resultados dependen en gran medida de que los programas psicoeducativos estén fundamentados en las características y necesidades familiares. ${ }^{16}$

Sin embargo, la mayoría de los estudios dedicados al análisis de la efectividad de la psicoeducación a familiares de pacientes con esquizofrenia ${ }^{21-26}$ se centran en analizar los cambios en la emotividad expresada y en la tasa de recaídas, rara vez evalúan la eficacia que tienen en el control de los síntomas, en especial los negativos. Los pocos estudios publicados que reflejan alguna efectividad de las intervenciones psicoeducativas sobre los síntomas negativos no incluyen un grupo control, evalúan grupos pequeños y sus criterios de inclusión son muy generales. ${ }^{27} \mathrm{La}$ escasez de investigaciones al respecto pudiera deberse a que la mayoría de los familiares de pacientes con esquizofrenia participan en el tratamiento del paciente cuando éste se encuentra hos- pitalizado, es decir cuando los síntomas positivos están exacerbados y constituyen el foco de atención del tratamiento.

\section{Entrenamiento en habilidades sociales}

El entrenamiento en habilidades sociales (EHS) para los pacientes con esquizofrenia se planteó debido a que los medicamentos antipsicóticos no mejoran directamente las habilidades necesarias para la vida en comunidad. Esta intervención es pertinente por muchas razones, entre las que destacan: 1. el aplanamiento afectivo obstaculiza la expresión social, 2. la mayoría de los pacientes presentan una historia de carencia de aprendizaje de estas habilidades, incluso antes de la aparición del trastorno y 3. frecuentemente existe carencia de estimulación ante el aislamiento social que generalmente acompaña a la enfermedad..$^{28}$

Entre los objetivos específicos del EHS se encuentran el desarrollo de asertividad, habilidades de conversación, control de la medicación, búsqueda de trabajo, habilidades recreativas, habilidades para hacer amigos, comunicación con la familia y solución de conflictos, ${ }^{28-33}$ mediante una combinación de técnicas que incluyen instrucciones focalizadas, modelado en video, ensayo conductual con retroalimentación inmediata, restructuración cognitiva y planificación de generalización, enfocadas a la reducción de la apatía, las dificultades de memoria y la incapacidad para solucionar problemas. ${ }^{34,35}$

Este tratamiento es uno de los más utilizados y evaluados en pacientes con esquizofrenia debido a su efectividad para lograr que el paciente adquiera y mantenga habilidades relacionadas con el funcionamiento comunitario independiente. $^{36}$

Algunos meta-análisis ${ }^{3,37}$ han concluido que mientras más conductuales son las evaluaciones mejores resultados refleja el EHS. Así, la mayoría de las investigaciones reportan una efectividad mayor en la adquisición de habilidades y asertividad que en otros padecimientos. En esta última variable no separan en el análisis del efecto los síntomas positivos y los negativos. Debido a esto, la evidencia de efectividad del EHS en los síntomas de esquizofrenia es escasa; en cuanto a los síntomas positivos, se ha reportado que el beneficio es reducido durante la fase activa de la terapia e incluso se pierde a medida que pasa el tiempo. ${ }^{38}$ Entre los pocos estudios que evalúan la efectividad de esta intervención en los síntomas negativos se encuentra el de Valencia et al. ${ }^{29}$ quienes reportan una disminución de la sintomatología negativa mediante programas de aprendizaje de habilidades sociales con duración de seis meses a un año. Por su parte, Lecomte, Leclerc y Wykes ${ }^{39}$ encontraron en su estudio que a pesar de que existió una disminución de la gravedad de los síntomas negativos al momento del postratamiento, estos resultados no se mantuvieron en el seguimiento a 12 meses.

Kurtz y Mueser ${ }^{40}$ realizaron un meta-análisis en el que el EHS mostró un tamaño del efecto mediano en cuanto a 
habilidades sociales, habilidades de la vida diaria, funcionamiento en su comunidad y síntomas negativos, y un tamaño del efecto pequeño respecto a los síntomas positivos, los cognitivos y la prevención de recaídas.

A lo largo de las tres últimas décadas, el EHS ha sido una técnica central para tratar el pobre funcionamiento psicosocial de los pacientes con esquizofrenia. ${ }^{41,42}$ Hoy en día, debido a su efectividad, diversas investigaciones continúan adaptando y perfeccionando la técnica con el fin de tener mejores resultados; por ejemplo, con el entrenamiento virtual para combatir el problema del ausentismo del paciente al tratamiento ${ }^{43}$ o con la combinación de este tipo de intervención psicosocial con otros tipos de terapia ${ }^{44}$ para mejorar los resultados en pacientes con predominio de síntomas negativos, quienes generalmente se muestran refractarios al EHS debido a que el deterioro cognitivo limita su potencial de aprendizaje. ${ }^{45}$

El hecho de que existan pocas investigaciones sobre el EHS que evalúan como principal variable de efecto los síntomas negativos puede deberse a que inicialmente este tipo de intervención psicosocial no fue conceptualizada para tratar los síntomas de la esquizofrenia sino para aumentar la funcionalidad e impulsar al paciente a una vida independiente. A pesar de la existencia de investigaciones que prueban la efectividad de esta terapia en la disminución de los síntomas negativos, es claro que el efecto no es grande y es de corta duración.

\section{Rehabilitación cognitiva}

La terapia de rehabilitación cognitiva se define como el entrenamiento conductual que tiene por objetivo mejorar los procesos cognitivos (atención, memoria, funciones ejecutivas, cognición social o metacognición) con la meta de alargar su duración y generalización. ${ }^{46}$ Se ha identificado que los pacientes con esquizofrenia presentan déficits cognitivos en la memoria declarativa verbal, las funciones ejecutivas, la atención sostenida y la memoria de trabajo. Estas alteraciones se asocian con los déficits en el funcionamiento social, ocupacional y en la capacidad de vivir independientemente. En esta dirección, se ha visto la necesidad de enfocar la práctica clínica de acuerdo con estas discapacidades por medio de la terapia orientada al deterioro cognitivo. ${ }^{47}$ La Rehabilitación Cognitiva cobra un gran auge a partir de la década de los años 1990 debido al surgimiento de investigadores que plantean los trastornos cognitivos de la esquizofrenia como puntos nodales del padecimiento. ${ }^{48}$

Existe suficiente evidencia empírica de que este tipo de intervención tiene efectos de medianos a grandes en la recuperación de las capacidades cognitivas de los pacientes; ${ }^{48-50}$ pues, en general, los estudios dirigidos a evaluar su efectividad se enfocan a variables como la atención, la memoria y el funcionamiento ejecutivo, ${ }^{51-53}$ es decir, a variables relacionadas con los procesos mentales básicos que generalmente se ven afectados.
Sin embargo, Pfammatter et al. ${ }^{3}$ reportan que entre 63 estudios que valoran la efectividad de la terapia de rehabilitación cognitiva, únicamente nueve evaluaron síntomas negativos y el tamaño del efecto en esta variable fue pequeño. Otros meta-análisis concluyen que a pesar de que se ha probado la efectividad de este tipo de intervención en la cognición social, el funcionamiento social y la psicopatología general, ${ }^{51-53}$ ésta es limitada y no es posible asegurar que los resultados se mantengan a través del tiempo.

Las escasas investigaciones que reflejan resultados positivos respecto a este tipo de intervención en los síntomas negativos tratan sobre la rehabilitación cognitiva asistida por computadora, ${ }^{54}$ pacientes de diagnóstico reciente, ${ }^{55}$ terapias psicológicas que integran más de una modalidad de intervención ${ }^{56,57}$ y rehabilitación neurocognitiva. ${ }^{58}$

En un estudio reciente, se compararon los efectos de la terapia de rehabilitación cognitiva y la TCC para la esquizofrenia; se concluyó que ambas tuvieron un efecto moderado en la reducción de los síntomas negativos y que no existieron diferencias significativas entre ambos tipos de intervención. ${ }^{59}$ Los resultados de este estudio invitan a la búsqueda de intervenciones que muestren un efecto mayor sobre los síntomas negativos y al análisis de los factores que intervienen en la efectividad de los diferentes tipos de psicoterapia para la esquizofrenia.

\section{Terapia cognitiva para síntomas positivos}

La terapia cognitiva para los síntomas positivos tiene como principal objetivo brindar herramientas para el manejo de las experiencias psicóticas, enseñando al paciente no solamente a reestructurar las creencias sobre la naturaleza de estas experiencias sino a dar sentido psicológico a sus síntomas positivos. ${ }^{60}$ Debido a la relación entre emociones negativas intensas y la exacerbación de síntomas psicóticos, la terapia cognitiva debe incluir un entrenamiento en control emocional basado en técnicas de relajación, respiración, detención del pensamiento y restructuración cognitiva de creencias asociadas a dichas emociones. ${ }^{61}$

Diferentes estudios muestran evidencia de la efectividad de la terapia cognitiva para la disminución de la gravedad de la sintomatología positiva, ${ }^{62-64}$ de la angustia producida por los delirios, ${ }^{62}$ del número de días hospitalizados ${ }^{63} \mathrm{e}$ incluso de síntomas de depresión. ${ }^{64}$

Empero, en una revisión sistemática ${ }^{65}$ de las investigaciones realizadas con este tipo de tratamiento psicosocial se concluye que, aunque esta intervención es efectiva para reducir síntomas positivos (incluso en pacientes con síntomas positivos resistentes al medicamento), ${ }^{66}$ tiene un efecto débil sobre los síntomas negativos y el funcionamiento social.

En cualquier caso, su potencial para disminuir la gravedad de los síntomas negativos ${ }^{64,67}$ debe evaluarse en ensayos clínicos con mayor rigor metodológico ${ }^{68}$ que estén dirigidos específicamente a determinar su efecto en los síntomas ne- 
gativos, pues los criterios de inclusión para evaluar la efectividad de esta intervención han sido restrictivos, en especial con los pacientes que presentan deterioro cognitivo, pobre funcionamiento social y predominio de síntomas negativos.

\section{Terapia cognitivo conductual orientada a la recuperación}

Ante la necesidad de evidencia empírica de la efectividad de la TCC en pacientes con predominio de sintomatología negativa, Grant et al. ${ }^{14}$ diseñaron y evaluaron la efectividad de una TCC para la esquizofrenia basada en el movimiento de recuperación y especializada en pacientes con bajo funcionamiento psicosocial y predominio de síntomas negativos; a esta terapia le denominan Terapia cognitivo conductual para pacientes con bajo funcionamiento o Terapia cognitivo conductual orientada a la recuperación (TCC-R).

El término recuperación ha sido controversial en la esquizofrenia debido a que algunos profesionales de la salud consideran este padecimiento como crónico con síntomas persistentes, recurrentes y debilitantes que eliminan la esperanza de una recuperación del funcionamiento psicosocial. Sin embargo, existen investigaciones que muestran que los factores que influyen en la recuperación son susceptibles de cambio mediante el tratamiento; es posible pues que los pacientes logren una remisión sostenida de los síntomas y mayores niveles de funcionamiento. ${ }^{69}$

Entre las características que debe cumplir un tratamiento orientado a la recuperación se encuentran: que el paciente determine sus propios objetivos y patrones individuales de recuperación (de acuerdo con sus características), apoyo en la toma de decisiones que tengan impacto en su vida, y un mensaje motivador en donde la recuperación sea vista como un continuo. ${ }^{70}$

La adaptación de la TCC-R (que originalmente tiene una duración de un año y medio) a una terapia conformada por 20 sesiones denominada Terapia Cognitivo Conductual para síntomas negativos (TCC-N), ${ }^{71,72}$ ha demostrado efectividad para reducir de forma notable la gravedad de los síntomas negativos y las creencias disfuncionales acerca de las habilidades, experiencias emocionales y socialización de los pacientes; no obstante, los autores señalan también que es necesario realizar nuevas investigaciones con pacientes control y con seguimientos a largo plazo.

\section{DISCUSIÓN Y CONCLUSIÓN}

Dentro de las aproximaciones psicoterapéuticas dirigidas a pacientes con esquizofrenia, la terapia cognitivo conductual es una de las que cuenta con mayor evidencia de efectividad, ${ }^{3,60-62}$ tanto para reducir los síntomas positivos y los negativos como para mejorar el funcionamiento general de los pacientes; sin embargo, a pesar de los avances que han presentado tanto los tratamientos farmacológicos como los psicosociales, los problemas generados por la sintomatología negativa de pacientes con diagnóstico de esquizofrenia están aún lejos de resolverse.

Esto puede deberse a que los síntomas negativos tienden a persistir en el tiempo ya que se asocian con déficits cognitivos, responden menos al tratamiento con neurolépticos, y la característica falta de energía convierte en un gran reto que el paciente acuda a psicoterapia.

La presente revisión confirma la escasez de estudios dedicados a evaluar específicamente la efectividad de las intervenciones psicosociales en los síntomas negativos de pacientes con esquizofrenia. ${ }^{64,67,68}$ La mayoría de los meta-análisis y revisiones al respecto ${ }^{4,6,68,71-73}$ señalan la necesidad de generar evidencia científica sólida en relación con el tratamiento de estos síntomas.

A pesar de que la TCC ha demostrado un tamaño del efecto mediano en el tratamiento de síntomas positivos, ${ }^{3}$ se han dejado de lado los pacientes con sintomatología negativa debido a que generalmente presentan déficits cognitivos. En el meta-análisis de Wykes, ${ }^{68}$ por ejemplo, sólo un estudio evaluó los síntomas negativos como principal variable dependiente del tratamiento, revelando un tamaño del efecto moderado en pacientes sin deterioro cognitivo importante.

Al comparar la escasa evidencia generada en este campo, es posible concluir que, entre las diferentes técnicas cognitivo conductuales para el tratamiento de la esquizofrenia, la Terapia Cognitivo Conductual orientada a la Recuperación (TCC-R) resulta la de mayor efecto sobre los síntomas negativos ${ }^{14,69}$ que tanto dificultan la vida independiente y el funcionamiento psicosocial de los pacientes. ${ }^{74} \mathrm{Su}$ implementación, en combinación con el tratamiento farmacológico y el psicosocial de la sintomatología positiva puede constituir un avance significativo en la atención que requieren y merecen las personas que padecen esquizofrenia.

\section{Financiamiento}

Ninguno.

\section{Conflicto de intereses}

Los autores declararon no tener conflicto de intereses.

\section{Agradecimientos}

El presente texto es parte del trabajo doctoral de Tania Morales Vigil, quien recibió una beca para el financiamiento de sus estudios en la Facultad de Psicología de la Universidad Nacional Autónoma de México, por parte del Consejo Nacional de Ciencia y Tecnología (CONACYT).

\section{REFERENCIAS}

1. Laughren T, Levin R. Food and Drug Administration perspective on negative symptoms in schizophrenia as a target for a drug treatment claim. Schizophr Bull 2006;32:220-222. 
2. Kurtz MM. Symptoms versus neurocognitive test performance as predictors of psychosocial status in schizophrenia: a 1- and 4-year prospective study. Schizophr Bull 2005;31:167-174.

3. Pfammatter M, Junghan U, Brenner $\mathbf{H}$. Efficacy of psychological therapy in schizophrenia: Conclusions from meta-analyses. Schizophrenia Bull 2006;32(1):64-80.

4. Slade $P$, Hadock $G$. A historical overview of psychological treatments for psychotic symptoms. En: Hadock G, Slade P (eds.). Cognitive-behavioural interventions with psychotic disorders. Londres: Routledge; 1996.

5. Kuipers E. The role of CBT in relapse prevention of schizophrenia. Schizophr Res 2012;136(1):58-59.

6. Kern R, Glynn S, Horan W, Marder S. Psychosocial treatments to promote functional recovery in schizophrenia. Schizophrenia Bull 2009;35(2):347-361.

7. Roder V, Müller D, Brenner H, Spaulding W. Integrated Psychological Therapy (IPT) for the treatment of neurocognition, social cognition and social competency in schizophrenia patients. Massachusetts: Hogrefe; 2011.

8. Tai S, Turkington D. The evolution of cognitive behavior therapy for schizophrenia: current practice and recent developments. Schizophrenia Bull 2009;35:865-873.

9. Xia J, Merinder L, Belgamwar M. Psychoeducation for schizophrenia. Schizophrenia Bull 2011;37(1):21-22.

10. Granholm E, Ben-Zeev D, Link P. Social disinterest attitudes and group cognitive-behavioral social skills training for functional disability in schizophrenia. Schizophrenia Bull 2009;35(5):874-883.

11. Redoblado M, Siciliano D, Withey $P$, Moss B et al. A randomized controlled trial of cognitive remediation in schizophrenia. Schizophrenia Bull 2010;36(2):419-427.

12. Brabban A, Tai S, Turkington D. Predictors of outcome in brief cognitive therapy for schizophrenia. Schizophrenia Bull 2009;35(5):859-864.

13. Chadwick P, Birchwood M, Trower P. Cognitive therapy for delusions, voices and paranoia. Inglaterra: Wiley; 1996.

14. Grant P, Huh G, Perivoliotis D, Stolar N et al. Randomized trial to evaluate the efficacy of cognitive therapy for low-functioning patients with schizophrenia. Arch Gen Psychiat 2012;69(2):121-127.

15. Woo S, Goldstein M, Nuechterlein K. Relatives' affecting style and the expression of subclinical psicopathology in patients with schizophrenia. Fam Process 2004;43:233-247.

16. Fresán A, Apiquián R, Ulloa R, Loyzaga C et al. Ambiente familiar y psicoeducación en el primer episodio de esquizofrenia: resultados preliminares. Salud Mental 2001;24(4):36-40.

17. Bebbington $P$, Kuipers L. The predictive utility of expressed emotion in schizophrenia: Aggregate analysis. Psychol Med 1994;24:707-718.

18. Weisman A. Integrating culturally-based aproachments with existing interventions for hispanic/latino families coping with schizophrenia. Psychotherapy: Theory, Research, Practice, Training 2005;42:178-197.

19. Vallina $O$, Lemos S, Fernández P. Estado actual de la detección e intervención temprana en psicosis. Apuntes Psicología 2006;24(1-3):185221.

20. Baüml J, Froböse T, Kraemer S, Rentrop M et al. Psychoeducation: a basic psychotherapeutic intervention for patients with schizophrenia and their families. Schizophrenia Bull 2006;32(1):1-9.

21. Sin J, Norman I. Psychoeducational interventions for family members of people with schizophrenia: a mixed-method systematic review. J Clin Psychiat 2013;74(12):1145-1162.

22. Leff J, Kuipers L, Berkowitz R, Sturgeon D. A controlled trial of social intervention in the families of schizophrenic patients. Brit J Psychiat 1985;146:594-600.

23. Hogarty G, Anderson C, Reiss D, Kornblith S et al. Family psychoeducation, social skills training, and maintenance chemotherapy in the aftercare treatment of schizophrenia. Arch Gen Psychiat 1991;48(4):340-347.

24. Dixon L, Adams C, Luckstead A. Update of family psicoeducation for schizophrenia. Schizophrenia Bull 2000;26:5-20.
25. Xiong W, Phillips M, Hu X, RuiWen W et al. Family based intervention of schizophrenic in China. A randomized control trial. Brit J Psychiat 1994;165:501-506.

26. Pharoah F, Mari J, Streiner D. Family intervention for schizophrenia. Oxford: The Cochrane Library; 2005.

27. Dyck DG, Short RA, Hendryx MS, Norell D et al. Management of negative symptoms among patients with schizophrenia attending multiple-family groups. Psychiat Serv 2000;51(4):513-519.

28. Mueser K. Tratamiento cognitivo-conductual de la esquizofrenia. En: Caballo V (dir.) Manual para el tratamiento cognitivo/conductual de los trastornos psicológicos. Vol. 1. Madrid: Siglo XXI; 1997.

29. Valencia M, Liberman R, Rascón M, Juárez F. Habilidades psicosociales para la esquizofrenia. En: Valencia M (comp.). Alternativas terapéuticas para la esquizofrenia. México: Herder; 2012; pp. 23-71.

30. Valencia M, Rascón M, Ortega-Soto H. El tratamiento psicosocial en los pacientes con esquizofrenia. En: Ortega-Soto $\mathrm{H}$, Valencia-Collazos M (eds.). Esquizofrenia. Estado actual y perspectivas. México: Publicaciones del Instituto Nacional de Psiquiatría Ramón de la Fuente Muñiz; 2001.

31. Valencia M, Rascón M, Quiroga H. Aportaciones de la investigación respecto al tratamiento psicosocial y familiar de pacientes con esquizofrenia. Salud Mental 2003;26(5):1-18.

32. Valencia M, Ortega-Soto H, Rodríguez M, Gómez L. Estudio comparativo de consideraciones clínicas y psicoterapéuticas en el tratamiento biopsicosocial de la esquizofrenia. Primera parte. Salud Mental 2004;27(3):47-53.

33. Valencia M, Díaz A, Juárez F. Integration of pharmacological and psychosocial treatment for schizophrenia in Mexico: The case of a developing country proposal. En: Badria F (ed.). Pharmacotherapy. EUA: InTech; 2012; pp. 41-69.

34. Bellack A, Mueser K, Gingerich S, Agresta J. Social skills training for schizophrenia: A step by step guide. Nueva York: Guilford Press; 1997.

35. Liberman R, DeRisi W, Mueser K. Social skills training for psychiatric patients: Psychology practitioners guidebooks. Nueva York: Pergamon Press; 1989.

36. Heinssen R, Liberman R, Kopelowikz R. Psychosocial skills training for schizophrenia: Lessons from laboratory. Schizophrenia Bull 2000;26:21-46.

37. Benton $\mathbf{M}$, Schroeder $\mathbf{H}$, Social skills training with schizophrenics. A meta-analytic evaluation. J Consult Clin Psych 1990;58:741-747.

38. Marder S, Wirshing W, Mintz J, McKenzie J et al. Two-year outcome of social skills training and group psychotherapy for outpatients with schizophrenia. Am J Psychiat 1996;153:1585-1592.

39. Lecomte T, Leclerc C, Wykes T. Group CBT for early psychosis-are there still benefits one year later? International J Group Psychotherapy 2012;62(2):309-321.

40. Kurtz M, Mueser K. A meta-analysis of controlled research on social skills training for schizophrenia. J Consult Clin Psych 2008;76:491-504.

41. Brady J. Social skills training for psychiatric patients, II: Clinical outcome studies. Am J Psychiat 1984;141:491-498.

42. Haldford W, Hayes R. Social skills training with schizophrenic patients. En: Kavanagh D (ed.). Schizophrenia: An overview and practical handbook. Londres: Chapman \& Hall; 1992.

43. Rus-Calafell M, Gutiérrez-Maldonado J, Ribas-Sabaté J. A virtual reality-integrated program for improving social skills in patients with schizophrenia: A pilot study. J Behav Ther Exp Psy 2014;45(1):81-89.

44. Elis O, Caponigro J, Kring A. Psychosocial treatments for negative symptoms in schizophrenia: Current practices and future directions. Clin Psychol Rev 2013;33:914-928.

45. Kopelowicz A, Wallace C, Zarate R. Teaching psychiatric inpatients to re-enter the comunity: A brief method of improving the continuity of care. Psychiatr Serv 1998;49:1313-1316.

46. Wykes T, Huddy V, Cellard C, McGurk S, Czobor P. A meta-analysis of cognitive remediation for schizophrenia: Methodology and effect sizes. Am J Psychiat 2011;168:472-485. 
47. Barrera A. Los trastornos cognitivos de la esquizofrenia. Rev Chil Neuropsi 2006;44(3):215-221.

48. Pardo V. Trastornos cognitivos en la esquizofrenia. Estudios cognitivos de pacientes esquizofrénicos puestos al día. Rev Psiquiat Urug 2005;69(1):71-83.

49. Hayes R, McGrath J. Cognitive rehabilitation for people with schizophrenia and related conditions. Oxford: The Cochrane Library; 2005.

50. Twamley E, Jeste D, Bellack A. A review of cognitive training in schizophrenia. Schizophrenia Bull 2003;29:359-382.

51. Grynszpan O, Perbal S, Pelissolo A, Fossati $P$ et al. Efficacy and specificity of computer-assisted cognitive remediation in schizophrenia: a meta-analytical study. Psychol Med 2011;41:163-173.

52. McGurk S, Twamley E, Sitzer D, Mchugo G et al. A meta-analysis of cognitive remediation in schizophrenia. Am J Psychiat 2007;164:17911802.

53. Krabbendam L, Aleman A. Cognitive rehabilitation in schizophrenia: a quantitive analysis of controlled studies. Psychopharmacology 2003;169:376-382.

54. Bellucci DM, Glaberman K, Haslam N. Computer-assisted cognitive rehabilitation reduces negative symptoms in the severely mentally ill. Schizophr Res 2003;59:225-232.

55. Eack SM, Greenwald DP, Hogarty SS et al. Cognitive enhancement therapy for early-course schizophrenia: effects of a two-year randomized controlled trial. Psychiatr Serv 2009;60:1468-1476.

56. Roder V, Mueller DR, Schmidt SJ. Effectiveness of integrated psychological therapy (IPT) for schizophrenia patients: a research update. Schizophr Bull 2011;37(Suppl 2):S71-S79.

57. Iwata K. Efficacy and application of cognitive rehabilitation: the importance of combining social skills training with cognitive rehabilitation. Psychiat Neurol Jap 2013;115(4):406-12.

58. Sánchez P, Peña J, Bengoetxea E, Ojeda $\mathrm{N}$ et al. Improvements in negative symptoms and functional outcome after a new generation cognitive remediation program: A randomized control trial. Schizophr Bull 2013; doi:10.1093/schbul/sbt057.

59. Klinberg S, Wölfgang W, Engel C, Wittorf A et al. Negative symptoms of schizophrenia as a target of cognitive behavioral therapy. Results of the randomized clinical tones study. Schizophrenia Bull 2011;37(2):98-110.

60. Yusupoff L, Haddock G, Sellwood W, Tarrier N. Cognitive behavioural therapy for hallucinations and delusions: current practices and future trends. En: Salkovskis $P$ (ed.). Trends in cognitive and behavioural therapies. Londres: Wiley; 1996; pp. 133-146.

61. Robles R, Paéz F, González J. Terapia cognitivo conductual para los trastornos psicóticos: fundamentos, evaluación y aplicación a propósi- to de un caso. En: Valencia M (comp.). Alternativas terapéuticas para la esquizofrenia. México: Herder; 2012; pp.331-361.

62. Kuipers E, Fowler D, Garety P, Chisholm D et al. London-east anglia randomised controlled trial of cognitive behavioural therapy for psychosis, III: follow-up and economic evaluation at 18 months. Brit J Psychiat 1998;173:61-68.

63. Tarrier N, Yusupoff L, Kinney C, McCarthy E et al. Randomised controlled trial of intensive cognitive behaviour therapy for patients with chronic schizophrenia. Brit Med J 1998;317:303-307.

64. Sensky $T$, Turkington $\mathrm{D}$, Kingdon $\mathrm{D}$, Scott $\mathrm{J}$ et al. A randomized controlled trial of cognitive-behavioral therapy for persistent symptoms in schizophrenia resistant to medication. Arch Gen Psychiat 2000;57:165-172.

65. Perona S, Cuevas C. Efectividad de la terapia cognitivo-conductual individual aplicada a los síntomas psicóticos. 1. Revisión de los diseños experimentales de caso único aplicado al tratamiento de las ideas delirantes. Aptes Psicol 1999;17(1 y 2):31-48.

66. Erickson DH. Cognitive-behaviour therapy for medication-resistant positive symptoms in early psychosis: a case series. Early Interv Psychiat 2010;4(3):251-256.

67. Rector N, Beck A. Cognitive behavioral therapy for schizophrenia: an empirical review. J Nerv Mental Dis 2001;189:278-287.

68. Wykes T, Steel C, Everitt B, Tarrier N. Cognitive behavior therapy for schizophrenia: effect sizes, clinical models, and methodological rigor. Schizophr Bull 2008;34(3):523-537.

69. Harding C, Zubin J, Strauss J. Chronicity in schizophrenia: revisited. Brit J Psychiat 1992;161(18):27-37.

70. Bellack A. Scientific and consumer models of recovery in schizophrenia: concordance, contrasts and implications. Schizophrenia Bull 2006;32(3):432-442.

71. Staring A, Huurne M, van der Gaag M. Cognitive behavioral therapy for negative symptoms (CBT-n) in psychotic disorders: A pilot study. Behav Ther Exp Psy 2013;44:300-306.

72. Turkington D, Morrison A. Cognitive therapy for negative symptoms of schizophrenia. Arch Gen Psychiat 2011;69(2):119-120.

73. Ellis O, Caponigro J, Kring A. Psychosocial treatments for negative symptoms in schizophrenia: Current practices and future directions. Clin Psychol Rev 2013;33:914-928.

74. Liberman R, Kopelwicz A. Un enfoque empírico de la recuperación de la esquizofrenia. Definir la recuperación e identificar los factores que pueden facilitarla. Rehabil Psicosoc 2004;1:12-29. 